\title{
Aquaporin 1 promotes the proliferation and migration of lung cancer cell in vitro
}

\author{
XIAOBAI WEI and JINGCHENG DONG \\ Department of Chinese Integrative Medicine, Huashan Hospital, Fudan University, Shanghai 200040, P.R. China
}

Received February 17, 2015; Accepted May 27, 2015

DOI: 10.3892/or.2015.4107

\begin{abstract}
To examine the potential role of aquaporin 1 (AQP1) in lung cancer progression, the effects of AQP1 expression and underlying mechanisms on cell proliferation and migration were investigated on LLC and LTEP-A2 cell lines in vitro. LLC and LTEP-A2 lung cancer cells with a discrepant AQP1 expression level were used to determine the role of AQP1 in cancer cell proliferation and migration potential. An immunofluorescence assay was used to detect AQP1 expression levels in the LLC and LTEP-A2 cell lines. The method targeting the knockdown of AQP1 on lung cancer cell lines by siRNA was established and validated by RT-PCR and western blot analysis. The proliferation and migration abilities of AQP1 knockdown cell lines were detected by MTT, invasion and wound-healing assays. Moreover, the alteration of MMP-2, MMP-9, TGF- $\beta$ and epidermal growth factor receptor (EGFR) expression, associated with the migration and metastasis potential of lung cancer cell lines, was identified by western blot analysis in transfected cells. In the tumor cell migration and invasion test, AQP1 knockdown significantly decreased the migration and invasion of AQP1-siRNA cells. Additionally, the expression levels of MMPs were markedly decreased after AQP1-siRNA treatment in the two cell lines. Moreover, the decrease of MMP-2/-9 expression on lung cancer cell lines was associated with AQP1-siRNA doses. However, AQP1 knockdown did not have a significant effect on TGF- $\beta$ and EGFR. The results suggest that AQP1 may facilitate lung cancer cell proliferation and migration in an MMP-2 and-9-dependent manner.
\end{abstract}

\section{Introduction}

Lung cancer is the leading cause of global cancer-related mortality, resulting in over a million deaths annually (1). Surgery, chemotherapy and radiotherapy are the major treatments for human non-small cell lung cancer (NSCLC).

Correspondence to: Professor Jingcheng Dong, Department of Chinese Integrative Medicine, Huashan Hospital, Fudan University, 12 Wulumuqi Mid Road, Shanghai 200040, P.R. China E-mail: jcdong2004@126.com

Key words: aquaporin 1, proliferation, migration, lung cancer, MMPs
However, the response rate of chemotherapy for patients with advanced NSCLC is only $20-35 \%$ with a median survival of 10-12 months $(2,3)$. Although light has been shed on the genomic mutation of human lung cancer $(4,5)$, the mechanisms involved in the proliferation and migration of lung cancer remain to be elucidated.

Aquaporins(AQPs)are afamily of small( $\sim 30 \mathrm{kDa} /$ monomer) membrane transport proteins that assemble in membranes as tetramers and act primarily as water-selective pores, facilitating osmotically driven water transport across cell plasma membranes $(6,7)$. It has been reported that AQP was expressed in a variety of human tumors, which in some cases was correlated with tumor grade (8-10). AQP has also been suggested to be of diagnostic and prognostic value $(11,12)$. Previous findings demonstrated that AQPs were involved in angiogenesis, tumor cell migration and proliferation (13-15). These results suggested that AQPs play a crucial role in tumor biology.

AQP1 is a member of the AQP family, and has been shown to be involved in cell migration in several cell types such as keratocytes (16), epithelial cells of the kidney (13), and gastric epithelial cells (17), B16F10 melanoma, and breast cancer cells (18). However, the function of AQP1 in lung cancer remains largely unclear. Whether AQP1 facilitates proliferation and the metastastic potential of lung cancer remains to be determined. In the present study, we successfully used RNA interference (RNAi) technology to silence the expression of AQP1 in lung adenocarcinoma LTEP-A2 and LLC cell lines. The in vitro experiments demonstrated that the proliferation and migration of cancer cells was reduced in AQP1-siRNA-transfected LTEP-A2 and LLC cells. In addition, AQP1 knockdown partly inhibited the proliferation and migration of lung cancer cells in MMP-2 and MMP-9-dependent manner.

\section{Materials and methods}

Cell culture. LLC and LTEP-A2 cell lines were cultured in RPMI-1640 supplemented with 10\% Hyclone fetal bovine serum (FBS; ThermoFisher Scientific, Fremont, CA, USA) in an atmosphere of $5 \% \mathrm{CO}_{2}$ at $37^{\circ} \mathrm{C}$. The cells were grown in $75-\mathrm{cm}^{2}$ culture flasks and harvested in a solution of trypsinEDTA at the logarithmic growth phase.

Specific siRNAs and transfection. One scrambled non-targeting siRNA (used for a negative control, mock) and one fluorescent siRNA were designed and produced by GenePharma Co., Ltd. 
(Shanghai, China). The sequences used were: SiAQP1, 5'-GACCCGCTCGGACTTACT-3' (sense) and 5'-CTTCTGG ACCCATGCTCT-3' (antisense); mock, 5'-UUCUCCGAACG UGUCACGUTT-3' (sense) and 5'-ACGUGACACGUUCGGA GAATT-3' (antisense). The 25-, 50- and 100-nM siRNAs were transfected into culture cells with Lipofectamine 2000 reagent (Invitrogen-Life Technologies, Carlsbad, CA, USA) according to the manufacturer's instructions. The cells were collected 24, 48,72 or $96 \mathrm{~h}$ after transfection for analysis. LTEP-A2 and LLC cells treated or untreated only with Lipofectamine 2000 reagent served as controls.

Reverse transcription-PCR. Total RNA was isolated from transfected cells using TRIzol reagent (Invitrogen-Life Technologies) according to the manufacturer's instructions. Briefly, $1 \mu \mathrm{g}$ total cell RNA was reverse-transcribed by a First Strand cDNA Synthesis kit (Amersham, Buckinghamshire, UK). Primers used for PCR amplification of AQP1 were: forward, 5'-CTT ACC TCC AGG ACC CTT CC-3' and reverse, 5'-TAG CTC ATC CAC ACG TGC TC-3', with an annealing temperature of $60^{\circ} \mathrm{C}$. The conditions used for PCR were: $94^{\circ} \mathrm{C}$ for $30 \mathrm{sec}, 58^{\circ} \mathrm{C}$ for $30 \mathrm{sec}, 72^{\circ} \mathrm{C}$ for $40 \mathrm{sec} ; 30$ cycles and $72^{\circ} \mathrm{C}$ for $5 \mathrm{~min}$ for the final extension. The PCR products were separated on $1 \%$ agarose gel, visualized under UV and photographed. The result was analyzed using Quantity One 4.6.2 software for the optical density (OD).

Cell proliferation assay. Cell proliferative activities were examined using CCK-8. LTEP-A2 and LLC cells were seeded in 96-well plates $\left(1 \times 10^{3}\right.$ cells/well). Following treatment, CCK-8 was added to each well according to the manufacturer's instructions and incubated for $4 \mathrm{~h}$ at $37^{\circ} \mathrm{C}$. The $\mathrm{OD}$ value of each well was measured using a microplate reader (Spectra Thermo, Mannedorf, Switzerland) with a test wavelength of $450 \mathrm{~nm}$.

Western blot analysis. Total protein was extracted from the cells using a RIPA kit (Pierce, Rockford, IL, USA). Protein was electrophoresed on a polyacrylamide gel and transferred to Hybond-C nitrocellulose membranes. The membranes were incubated with anti-AQP1 (Chemicon, Temecula, CA, USA) and MMP-2, MMP-9, TGF- $\beta$ and epidermal growth factor receptor (EGFR) (all from Cell Signaling Technology) at $1: 1,000$ dilution at $37^{\circ} \mathrm{C}$ for $2 \mathrm{~h}$, and then with peroxidase-conjugated goat anti-rabbit IgG at room temperature for $1 \mathrm{~h}$. GAPDH was used as an internal control. Protein was visualized using enhanced chemiluminescence (ECL) methods. The membranes were washed three times and then exposed to $\mathrm{X}$-ray film. Western blot analysis and quantification analysis of blots were performed as previously described (19).

Immunofluorescence. The cells were fixed in $4 \%$ paraformaldehyde and embedded in paraffin or OCT for paraffin or frozen sections, respectively. Immunofluorescence (IF) staining of cells was performed as previously described (18). Anti-AQP1 primary antibody (1:100; Chemicon) and rabbit anti-mouse IgG antibody conjugated to Cy3 (1:100; Molecular Probes, Eugene, OR, USA) were used for IF staining.

Tumor cell migration and invasion assays. Assays to measure tumor cell migration were performed in a modified Boyden chamber (Transwell; Corning Costar, Tewksbury, MA, USA) containing a gelatin-coated polycarbonate membrane filter (8- $\mu \mathrm{m}$ pore size). BioCoat Matrigel (BD Biosciences, Bedford, MA, USA), which reconstitutes the basal membrane, was used to assess cell invasion. The degree of tumor cell migration and invasion was evaluated according to the manufacturer's instructions (25). Non-migrated cells were removed from the upper side of the membrane by scrubbing and migrated cells from the lower side of the membrane. Cell counting was accomplished by Coomassie blue staining and the cells were visualized under a microscope (Leica, Germany).

Wound-healing assay. Cells were cultured to a confluent monolayer, synchronized in 1\% FBS for $24 \mathrm{~h}$, and wounded by removing $\sim 400-\mu \mathrm{m}$ wide strips of cells across the well with a standard 200- $\mu$ l pipette tip. Wounded monolayers were washed twice with PBS to remove non-adherent cells. Wound healing was quantified by Image Plus software as a mean percentage of the remaining cell-free area, compared with the initial wound area (18).

Statistical analysis. Statistical analysis was performed using SPSS 10.0 software. Values are presented as mean \pm SD . Statistical analysis was performed using one-way analysis of variance (ANOVA) and the LSD post-hoc multiple comparison test. $\mathrm{P}$-values were two-sided and differences at $\mathrm{P}<0.05$ were considered to indicate statistical significance.

\section{Results}

Localization of AQP1 in LTEP-A2 and LLC cells. The AQP1 expression and localization of LTEP-A2 and LLC cells was investigated by immunofluorescence microscopy and western blot analysis. Western blot analysis revealed that AQP1 was expressed in LLC and LTEP-A2 cell lines (Fig. 1A). Compared to the LTEP-A2 cell line, the expression of AQP1 was more prominent in the LLC cell line. Fig. 1B shows that AQP1 (red) was detected in the cytosol and membrane in the two cell lines. DAPI was used to stain the nucleus.

Effective downregulation of AQP1 using siRNA in LTEP-A2 and LLC cells. LTEP-A2 and LLC cells were transfected with AQP1-siRNA and scrambled control siRNA. Treatment of the cells with AQP1-targeting siRNA (25,50 and $100 \mathrm{nM} \mathrm{SiAQP1)}$ and the scrambled siRNA (mock, $100 \mathrm{nM}$ ) for $96 \mathrm{~h}$ resulted in a significant downregulation of the AQP1 mRNA level and protein expression of AQP1 at $96 \mathrm{~h}$ following transfection with AQP1-siRNA, as determined by RT-PCR (Fig. 2A) and western blot analysis (Fig. 2B). As shown in Fig. 2 , the mock did not affect the expression levels of AQP1. However, compared with the mock, the expression of AQP1 in the 25, 50 and $100 \mathrm{nM}$ siRNA groups decreased at the protein and mRNA levels. Specifically, $100 \mathrm{nM}$ siRNA indicated an 80 and $85 \%$ reduction of AQP1 expression in LTEP-A2 and LLC cells, respectively. At the same time, there was minimal expression of AQP1 (red) in the cells transfected with AQP1siRNA, whereas the expression of AQP1 was not altered in the control cells by immunofluorescence analysis (Fig. 3). The results demonstrated that the AQP1-targeting siRNA was efficient to knock down the expression of AQP1 in LTEP-A2 and 


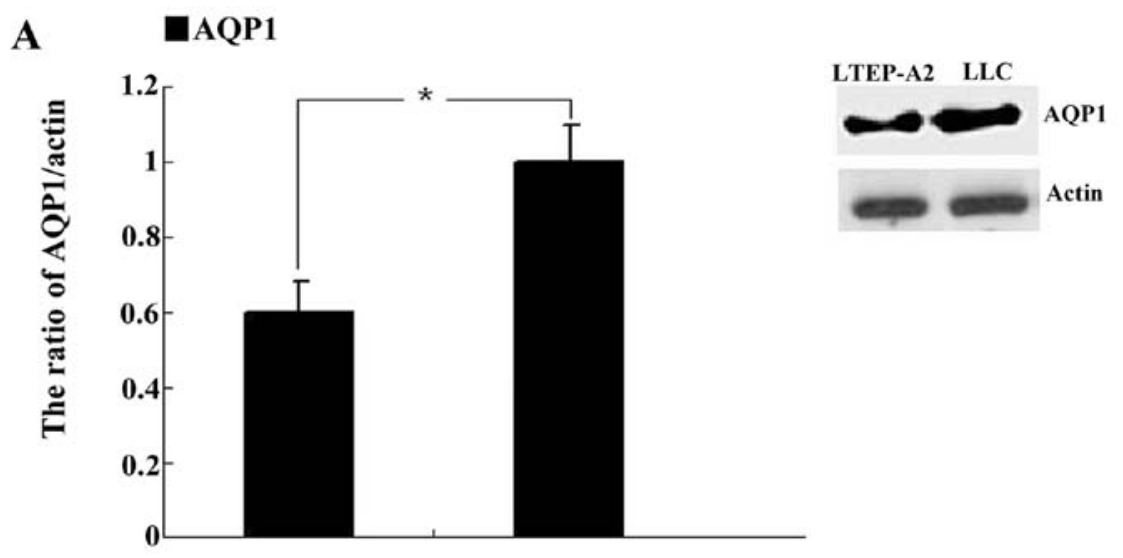

B

AQP1 expression in LLC and LTEP-A2 cells
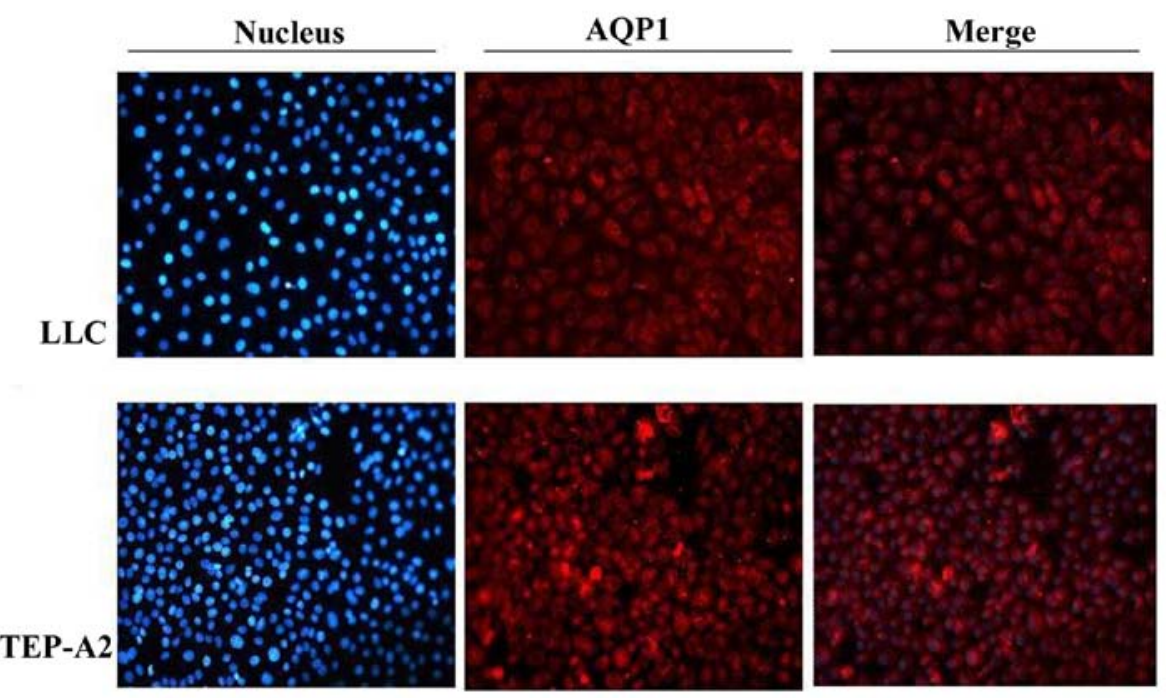

Figure 1. The expression of AQP1 in the LTEP-A2 and LLC cell lines by immunofluorescence localization and western blot analysis. (A) Expression of AQP1 in the LTEP-A2 and LLC cell lines by western blot analysis. When compared to the LTEP-A2 cell line, the expression of AQP1 is more prominent on LLC cell line. (B) Expression of AQP1 in the LTEP-A2 and LLC cell lines by immunofluorescence localization analysis. AQP1 (red) was detected in the cytosol and membrane in the two cell lines. DAPI was used to stain the nucleus. AQP1, aquaporin 1 . "P<0.05, compared with the control.

LLC cells. This downregulation was determined by western blot analysis using AQP1 antibody and the experiments were repeated three times $(n=3)$.

Effects of AQP1 downregulation on LTEP-A2 and LLC migration and proliferation. A significant downregulation in the AQP1 mRNA and protein level occurred at $96 \mathrm{~h}$, and $100 \mathrm{nM}$ siRNA groups exhibited the largest reduction of AQP1 expression in the LLC and LTEP-A2 cells, compared with the negative control $(\mathrm{P}<0.01$, Fig. 2$)$. siRNA groups $(100 \mathrm{nM})$ were thus selected to investigate whether a decreased expression of AQP1 affected cell migration and invasion. Fig. 4A shows that, AQP1 downregulation by siRNA resulted in decreased cell migration at $6 \mathrm{~h}$ and reduced tumor cell invasion at $24 \mathrm{~h}$. Moreover, the wound-healing assay showed significantly decreased wound closure in the AQP1 gene-silencing group vs. the mock group after 24-h scratching (Fig. 4B and C). The assays described above were performed in the presence of $0.04 \%$ mitomycin $\mathrm{C}$, an inhibitor of cell proliferation, and similar results were obtained. To determine the effect of AQP1 downregulation on cell proliferation, a CCK-8 assay was performed. A significant decrease in cell proliferation was observed following AQP1 downregulation as determined by the CCK-8 proliferation assay (Fig. 4C). There was a significant decrease in cell proliferation after 48 and $72 \mathrm{~h}$ following transfection with AQP1-siRNA.

Molecular mechanisms of the antitumor effects by $A Q P 1$-siRNA. The protein from the transfected cells was extracted to examine the effects of AQP1-siRNA on certain cytokines and signaling molecules. After $96 \mathrm{~h}$ of transfection and treatment with high concentrations of 50 and $100 \mathrm{nM}$ siRNA, the protein relative expression levels of MMP-2/-9 were decreased in LTEP-A2 and LLC cells, whereas no differences were identified in the control and mock groups (Fig. 5A-F). Furthermore, it was found that the higher the doses of siRNA, the lower the level of MMP-2/-9 expression. The expression of MMP-2/-9 was highest in the $25 \mathrm{nM}$ siRNA group, intermediate in the $50 \mathrm{nM}$ siRNA group, and lowest in the $100 \mathrm{nM}$ siRNA group. However, compared to the control and mock groups, transfection with high concentrations of 50 and $100 \mathrm{nM}$ AQP1-siRNA did not suppress the expression 
Time after transfection (h)

A LTEP-A2 $\frac{\text { LTEP-A2+AQP1-siRNA }}{\text { Control Mock } 25 \mathrm{nM} 50 \mathrm{nM} 100 \mathrm{nM}}$
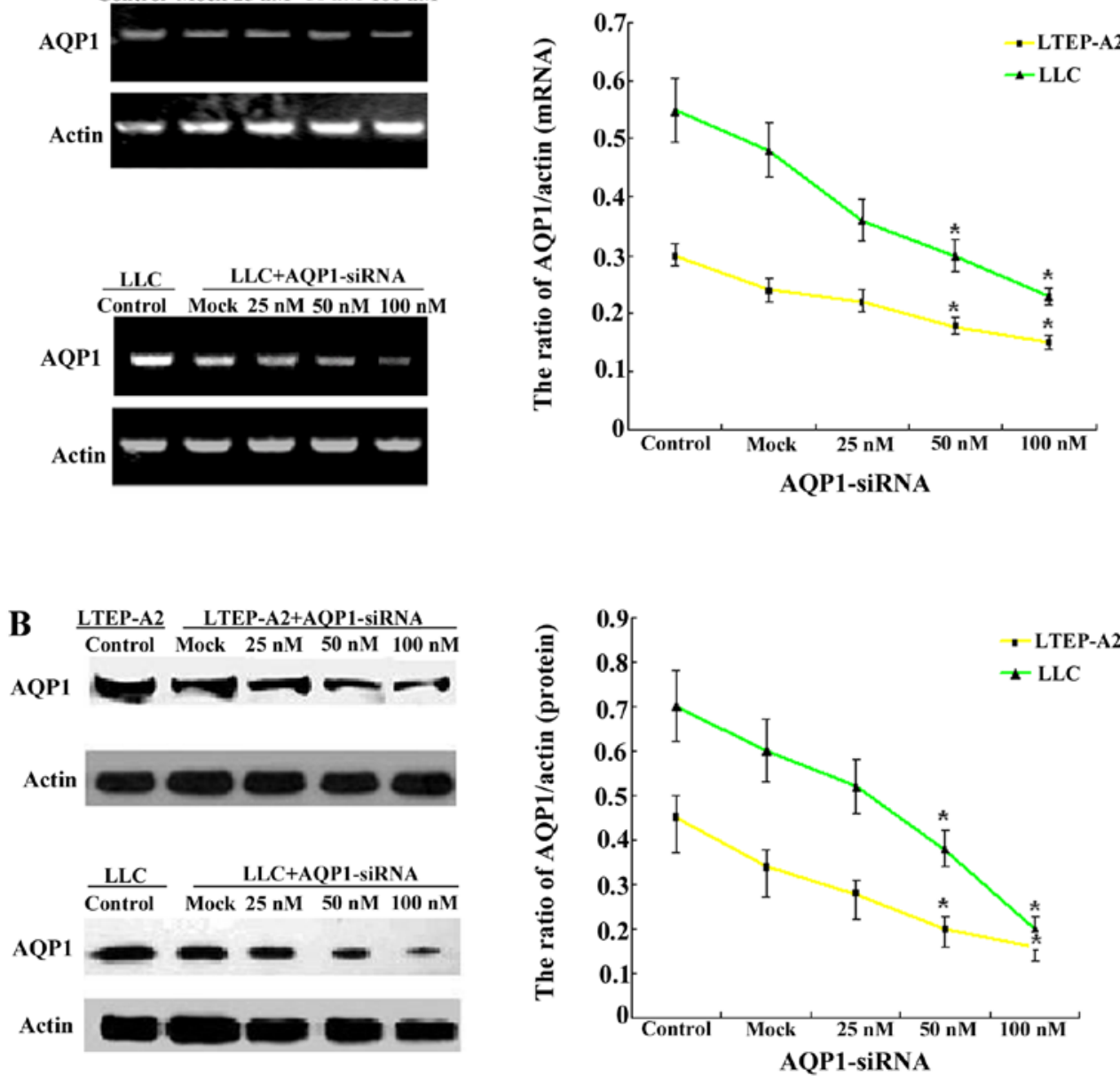

Figure 2. Effective silencing of AQP1 using siRNA. Downregulation of AQP1 expression in LTEP-A2 and LLC cell lines using siRNA as determined by (A) RT-PCR and (B) western blot analysis. AQP1, aquaporin 1."P<0.05, compared with the control.
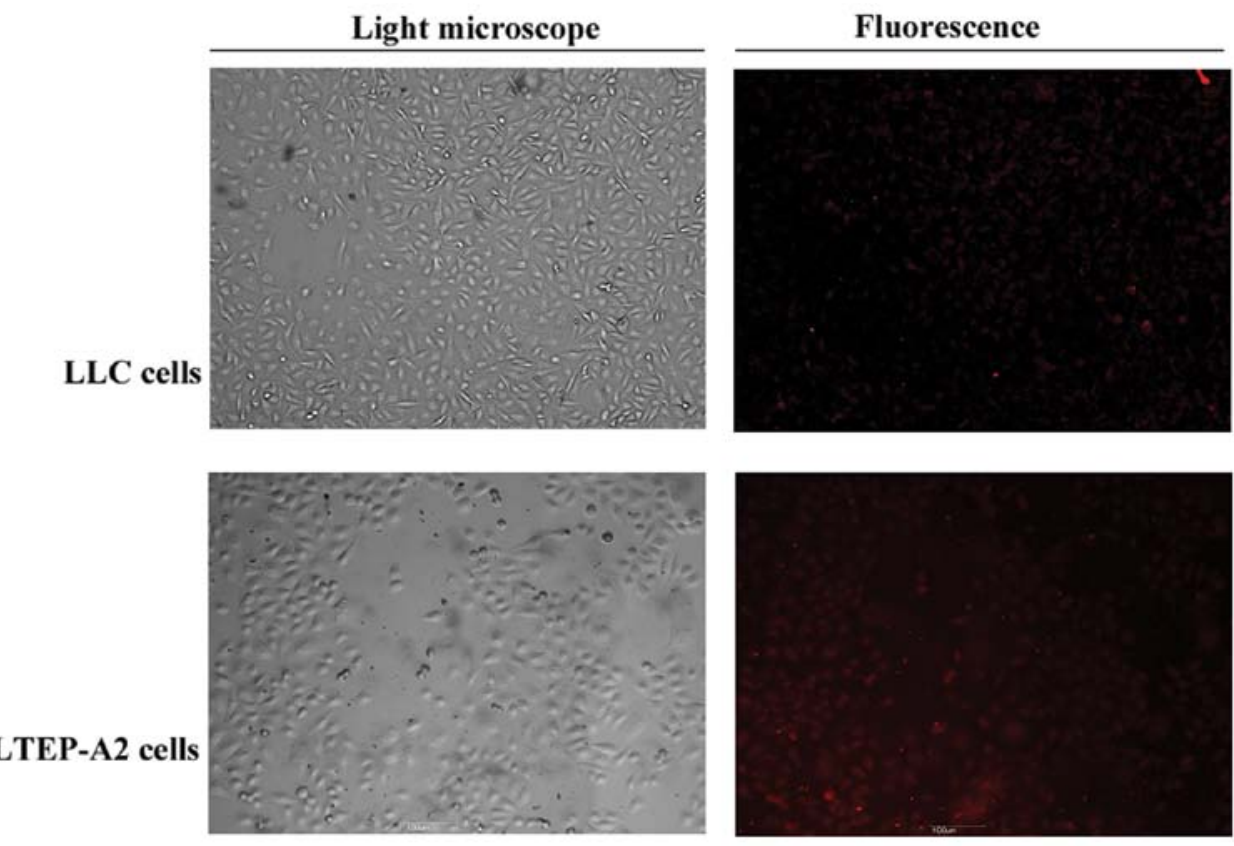

Figure 3. AQP1 expression in LLC and LTEP-A2 cells after siRNA transfection. Effective silencing of AQP1 using siRNA after $96 \mathrm{~h}$ post-transfection by immunofluorescence analysis. AQP1, aquaporin 1. 
A
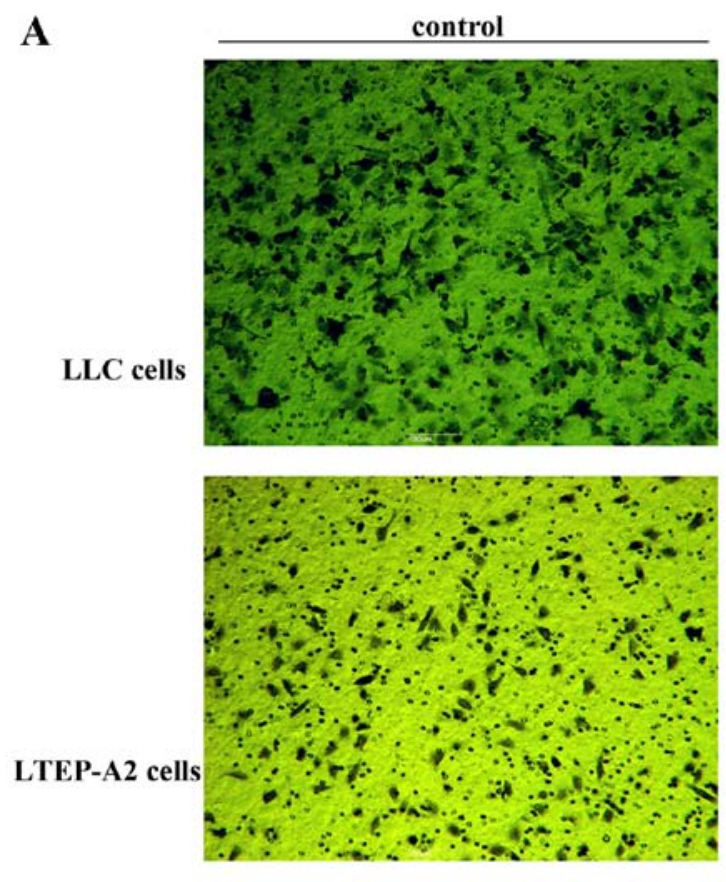

AQP1 - siRNA
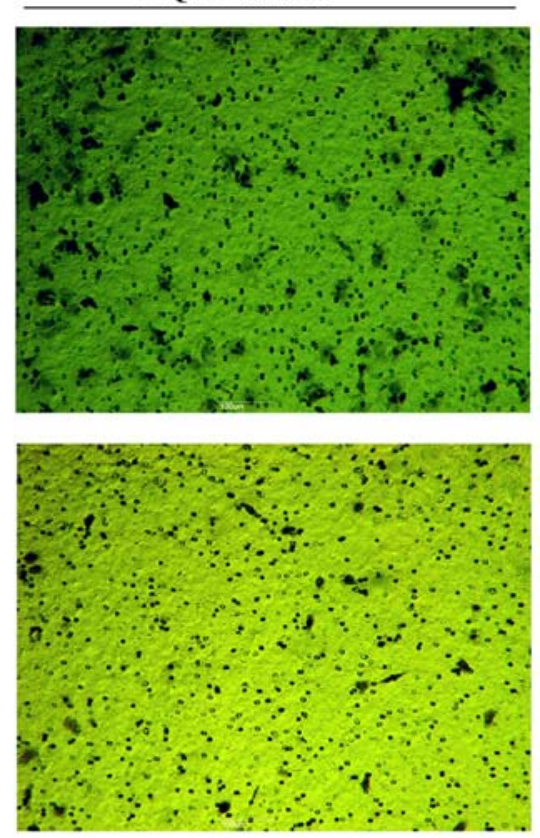

Deontrol

- AQP1- siRNA
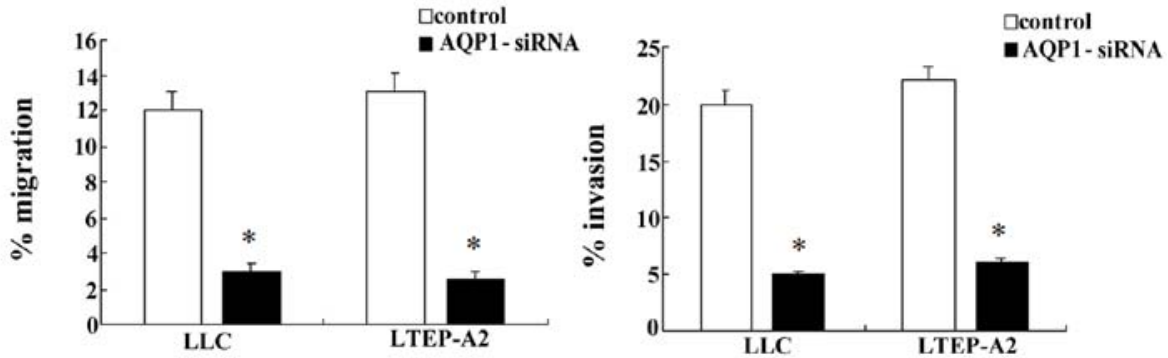

Figure 4. AQP1 gene silencing shows decreased migration and invasion potential in cells. (A) Summary of percentage of cell migration across nude Transwell membrane at $6 \mathrm{~h}$ and percentage of cell invasion through Matrigel-coated Transwell membranes at $24 \mathrm{~h}$. The results showed decreased cell migration and invasion in the AQP1 gene silencing in the LLC and LTEP-A2 cell line groups, compared with the control group $\left(\mathrm{n}=6\right.$; $\left.{ }^{*} \mathrm{P}<0.05\right)$.

of TGF- $\beta$ and EGFR (Fig. 5G-L). These data demonstrated that the knockdown of AQP1 by siRNA may inhibit MMP-2/-9, which are important in tumor progression.

\section{Discussion}

Despite advances in medical and surgical treatments, lung cancer remains the worldwide leading cause of cancer mortality (20). Due to intrinsic properties of lung adenocarcinoma in which cells show a high ability to progress rapidly, it has a poor prognosis in main histological types of lung cancer $(21,22)$. Therefore, how to effectively inhibit the proliferative and metastatic biological behavior of lung adenocarcinoma cells is a key issue to be overcome in order to improve the outcome.

As mentioned earlier, AQP1 has been found in tumors of different tissues, such as endometrioid adenocarcinoma and colon and breast cancer cells (23-25). Additionally, AQP1 expression in these tumor cells has been correlated with its metastatic potential $(26,27)$. For example, Hu and Verkman found that AQP1 facilitated tumor cell migration and extra invasion across blood vessels in B16F10 melanoma cells (18). However, the roles of AQP1 in oncogenesis and metastasis have not yet to be clearly defined in lung cancer.
In the present study, we first used LTEP-A2 and LLC cell lines to investigate the localization of AQP1. Previous findings on localization of AQP1 have shown that AQP1 is localized in the cytosol and cell membrane, which is in concordance with those of a previous study (28). Our results demonstrate that the localization of AQP1 in the cytosol and membrane may be cell-specific or a purely in vitro phenomenon. Furthermore, we have shown that chemically synthesized siRNAs specifically targeting AQP1 successfully knocked down the expression of AQP1 at the protein and mRNA levels from 80 to $85 \%$ in human lung adenocarcinoma LTEP-A2 cells and mouse lung adenocarcinoma LLC cells. The assays as described above were used to detect the effects on the biological behavior of LTEP-A2 and LLC cells in vitro. Using the CCK-8 assay, we were able to first show that the proliferation of AQP1-siRNA-transfected lung adenocarcinoma cells was significantly inhibited in vitro, although previous studies have failed to show this finding in colorectal cancer and B16F10 melanoma cells (29-31). We hypothesized that the main reason for inhibition of proliferation in lung adenocarcinoma cells by AQP1-siRNA is that AQP1 has distinct roles in different cell lines and tissues.

The formation of metastases is the leading cause of mortality in cancer patients. Establishment of metastasis is 
B

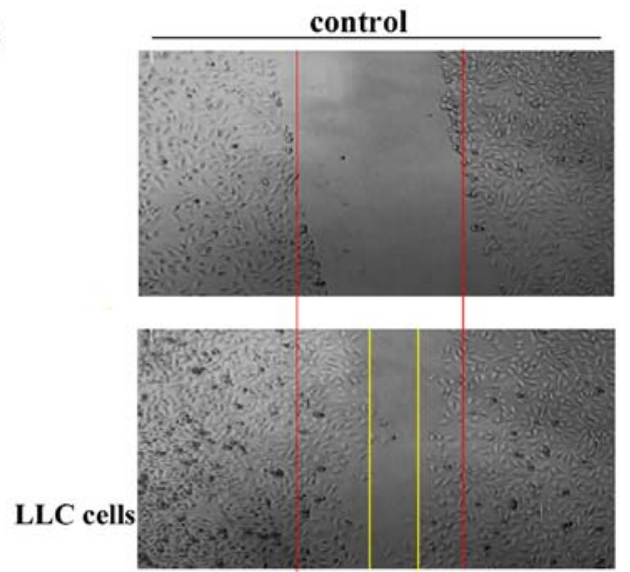

$0 \mathrm{~h} \longleftarrow 24 \mathrm{~h}$
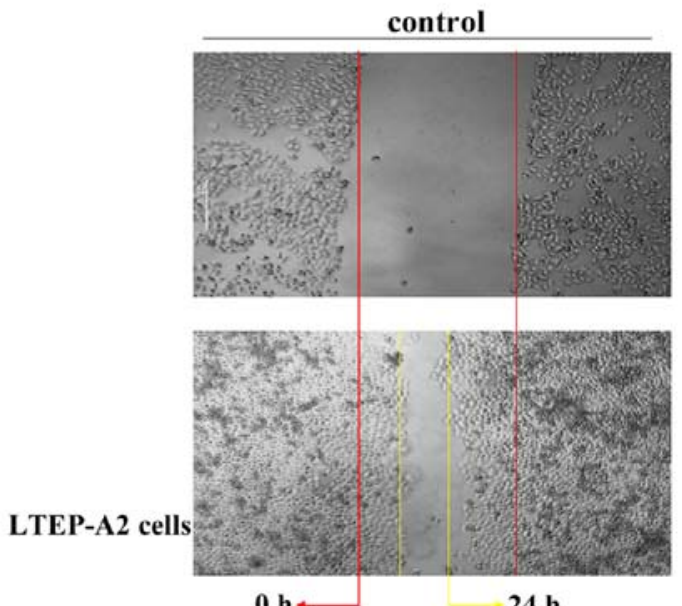

$0 \mathrm{~h} \longleftarrow 24 \mathrm{~h}$
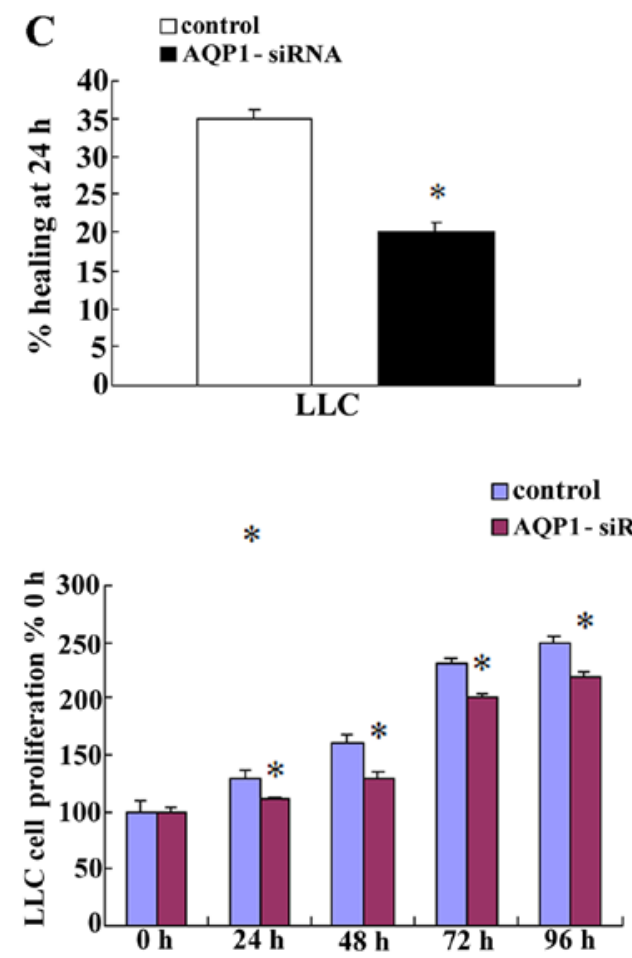
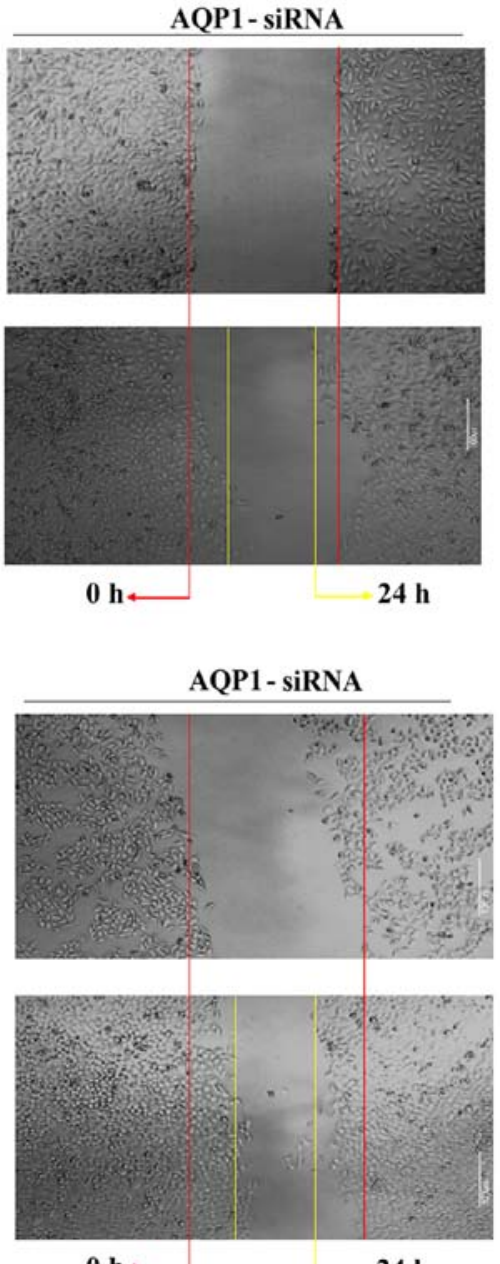

$0 \mathrm{~h} \longleftarrow 24 \mathrm{~h}$
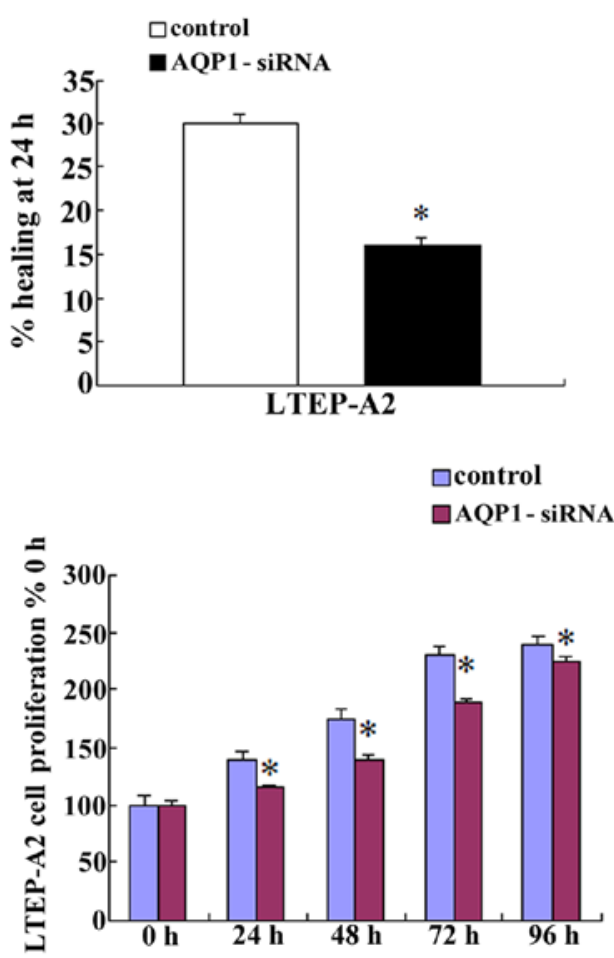

Figure 4. Continued. AQP1 gene silencing shows decreased migration and invasion potential in cells. (B) Summary of wound-healing assay associated with tumor cell migration. The scratched monolayer of the control and AQP1 gene-silencing group in LTEP-A2 and LLC cell lines at 0 and $24 \mathrm{~h}$ after scratching $\left(\mathrm{n}=6\right.$; $\left.{ }^{\mathrm{P}} \mathrm{P}<0.01\right)$. (C) Effect of AQP1 gene silencing on proliferation in LTEP-A2 and LLC cell lines. Cell viability was estimated using the CCK-8 assay and results showed decreased cell viability in the AQP1 gene-silencing groups. $\left(\mathrm{n}=6\right.$; $\left.{ }^{*} \mathrm{P}<0.05\right)$. AQP1, aquaporin 1. 
A

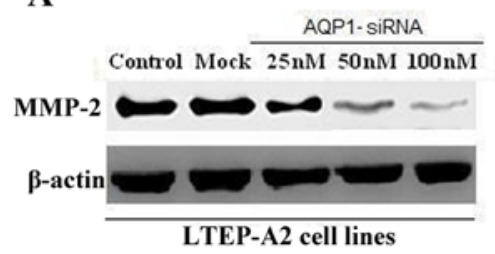

D

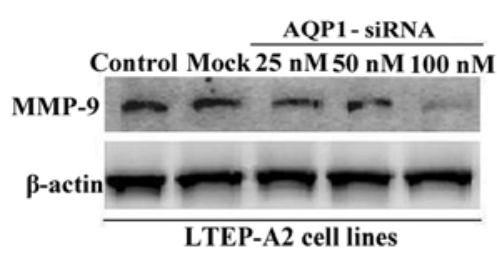

G

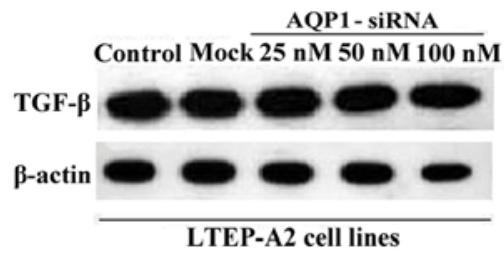

$\mathbf{J}$

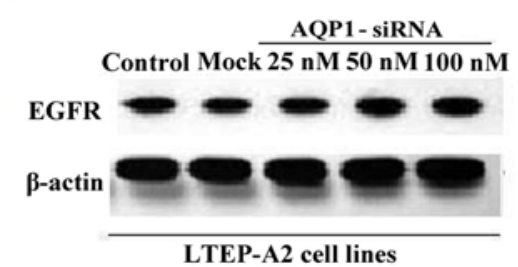

B

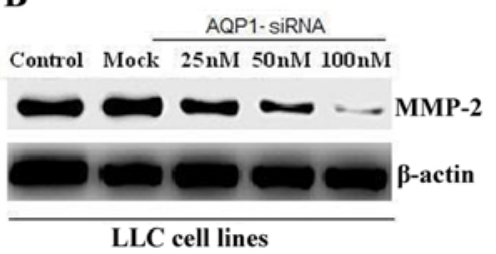

E

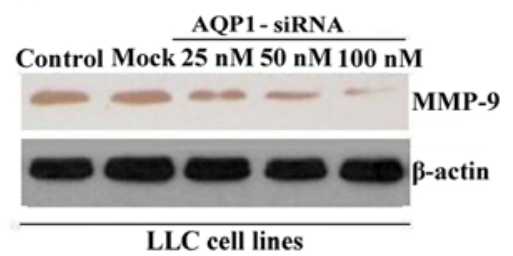

H

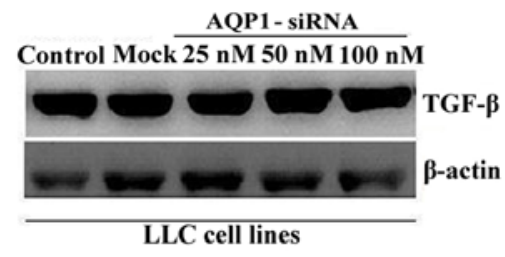

$\mathbf{K}$

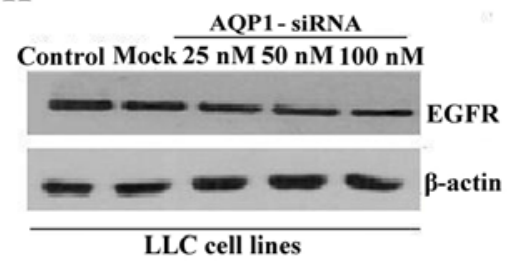

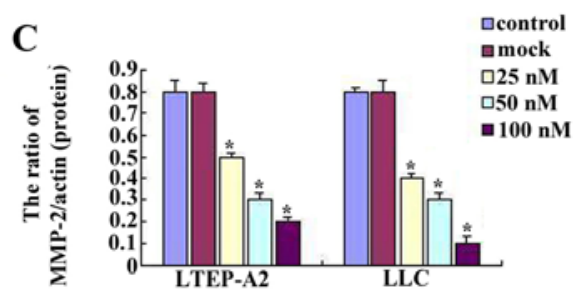
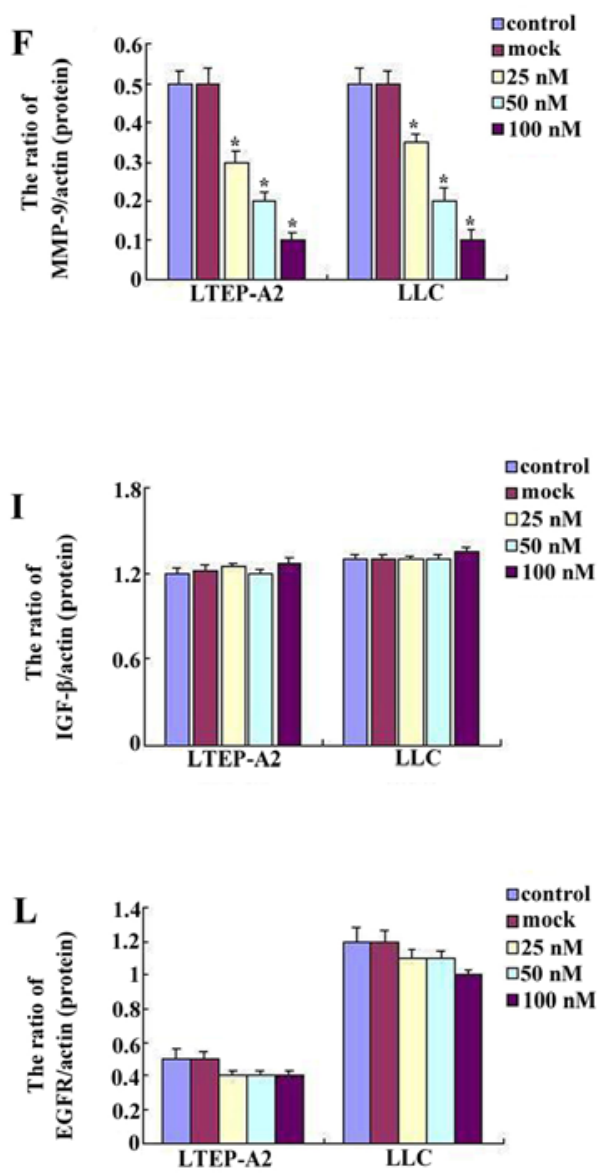

Figure 5. Western blot analysis of MMP-2/MMP-9/TGF- $\beta$ /EGFR after treatment of LTEP-A2 and LLC cell lines with AQP1-siRNA. (A-F) Representative images show that the 50 and $100 \mathrm{nM}$ AQP1-siRNA groups suppressed the MMP-2/-9 expression $\left(\mathrm{n}=6\right.$; $\left.^{*} \mathrm{P}<0.05\right)$. (G-L) Representative images show that there were no changes in the TGF- $\beta$ /EGFR expression after treatment with AQP1-siRNA in any siRNA dose ( $n=6$; $\left.{ }^{*} \mathrm{P}<0.05\right)$. AQP1, aquaporin 1 ; EGFR, epidermal growth factor receptor.

a multistep process involving the dissociation of cancer cells from the primary tumor, invasion of extracellular matrix, angiogenesis, intravasation into the vasculature or lymphatic systems, survival at these sites, extravasation and proliferation at a distant site (32-34). Downregulation of AQP1 has been shown to be involved in decreased cell migration in several cell types including human melanoma cell lines and human microvascular endothelial, kidney epithelial and gastric epithelial cells. Overexpression of AQP1 has been reported in proliferating tumor vessels suggesting its involvement in tumor angiogenesis (15). In the present study, the downregulation of AQP1 in LTEP-A2 and LLC cells by AQP1 gene silencing showed significantly decreased migration and invasion. These in vitro studies provide evidence that AQP1 is important in the development of lung cancer. However, we did not observe any significant changes in cell size and morphology following AQP1 downregulation in the LTEP-A2 and LLC cells.

AQP1 has emerged as an important player in metastasis. However, previous studies do not provide information regarding the downstream proteins induced by the inhibition of AQP1 expression via AQP1-siRNA in lung cancer or other cancer cells. Several MMPs are believed to be important in the process of angiogenesis, especially MMP-2 and MMP-9. These two MMPs are involved in lung cancer initiation, invasion, angiogenesis and metastasis (35-37).

TGF- $\beta$ and EGFR are crucial in many tumors. TGF- $\beta$ supports tumor progression by stimulating the transdifferentiation of epithelial cancer cells into migratory mesenchymal cells $(38,39)$ by promoting cell invasion, and dissemination to distant sites (40), and enhancing angiogenesis (41). EGFR is overexpressed in many epithelial-derived tumors and its 
role in the migration and survival of NSCLC is widely documented (42-44).

Since migration and invasion alter following treatment with AQP1-siRNA in lung cancer, whether changes of MMPs, TGF- $\beta$ and EGFR would occur was determined. Our results show that MMPs decreased after AQP1-siRNA in the two cell lines. It was found that the higher the doses of AQP1-siRNA, the lower the level of MMP-2/-9 expression. The expression of MMP-2/-9 was highest in the $25 \mathrm{nM}$ siRNA group, intermediate in the $50 \mathrm{nM}$ siRNA group and lowest in the $100 \mathrm{nM}$ siRNA group. However, AQP1 knockdown did not have a significant effect on the TGF- $\beta$ and EGFR, a finding that was consistent with results obtained by Zhang et al (45). These data indicate that AQP1 may produce a marked effect on tumor growth, vascularization and metastasis in lung adenocarcinoma by inhibiting MMP-2/-9, which play an important role in tumor progression.

In summary, to the best of our knowledge, we are first to report that the ability of lung adenocarcinoma LTEP-A2 and LLC cell line growth and metastasis is reduced after treatment with AQP1-siRNA. In addition, the expression of MMP protein decreased after LTEP-A2 and LLC cells were transfected with AQP1-siRNA, whereas no changes were observed for TGF- $\beta$ and EGFR. Our finidngs may therefore provide evidence for genetic therapy for lung adenocarcinoma.

\section{Acknowledgements}

This study was supported by the National Natural Science Foundation of China (no. 81173390/H2902), the National Basic Science Program of China (no.2009CB523000) and the Shanghai Science and Technology Committee (no. 09XD1400700).

\section{References}

1. Sun S, Schiller JH and Gazdar AF: Lung cancer in never smokers - a different disease. Nat Rev Cancer 7: 778-790, 2007.

2. Spiro SG and Silvestri GA: One hundred years of lung cancer. Am J Respir Crit Care Med 172: 523-529, 2005.

3. Spiro SG, Tanner NT, Silvestri GA, Janes SM, Lim E, Vansteenkiste JF and Pirker R: Lung cancer: Progress in diagnosis, staging and therapy. Respirology 15: 44-50, 2010.

4. Cancer Genome Atlas Research Network: Comprehensive genomic characterization of squamous cell lung cancers. Nature 489: 519-525, 2012.

5. Cancer Genome Atlas Research Network: Comprehensive molecular profiling of lung adenocarcinoma. Nature 511: 543-550, 2014.

6. Agre P, King LS, Yasui M, Guggino WB, Ottersen OP, Fujiyoshi Y, Engel A and Nielsen S: Aquaporin water channels - from atomic structure to clinical medicine. J Physiol 542: 3-16, 2002.

7. Verkman AS and Mitra AK: Structure and function of aquaporin water channels. Am J Physiol Renal Physiol 278: F13-F28, 2000.

8. Mobasheri A, Airley R, Hewitt SM and Marples D: Heterogeneous expression of the aquaporin 1 (AQP1) water channel in tumors of the prostate, breast, ovary, colon and lung: A study using high density multiple human tumor tissue microarrays. Int J Oncol 26 : 1149-1158, 2005.

9. Moon C, Soria JC, Jang SJ, Lee J, Obaidul Hoque M, Sibony M, Trink B, Chang YS, Sidransky D and Mao L: Involvement of aquaporins in colorectal carcinogenesis. Oncogene 22: 6699-6703, 2003 .

10. Saadoun S, Papadopoulos MC,Davies DC, Krishna S and Bell BA: Aquaporin-4 expression is increased in oedematous human brain tumours. J Neurol Neurosurg Psychiatry 72: 262-265, 2002.

11. Kafé H, Verbavatz JM, Cochand-Priollet B, Castagnet P and Vieillefond A: Collecting duct carcinoma: An entity to be redefined? Virchows Arch 445: 637-640, 2004.
12. Mazal PR, Stichenwirth M, Koller A, Blach S, Haitel A and Susani M: Expression of aquaporins and PAX-2 compared to CD10 and cytokeratin 7 in renal neoplasms: A tissue microarray study. Mod Pathol 18: 535-540, 2005.

13. Hara-Chikuma M and Verkman AS: Aquaporin-1 facilitates epithelial cell migration in kidney proximal tubule. J Am Soc Nephrol 17: 39-45, 2006.

14. Hara-Chikuma M and Verkman AS: Aquaporin-3 facilitates epidermal cell migration and proliferation during wound healing. J Mol Med (Berl) 86: 221-231, 2008.

15. Saadoun S, Papadopoulos MC, Hara-Chikuma M and Verkman AS: Impairment of angiogenesis and cell migration by targeted aquaporin-1 gene disruption. Nature 434: 786-792, 2005.

16. Ruiz-Ederra J and Verkman AS: Aquaporin-1-facilitated keratocyte migration in cell culture and in vivo corneal wound healing models. Exp Eye Res 89: 159-165, 2009.

17. Hayashi S, Takahashi N, Kurata N, Yamaguchi A, Matsui H, Kato $\mathrm{S}$ and Takeuchi $\mathrm{K}$ : Involvement of aquaporin-1 in gastric epithelial cell migration during wound repair. Biochem Biophys Res Commun 386: 483-487, 2009.

18. $\mathrm{Hu} \mathrm{J}$ and Verkman AS: Increased migration and metastatic potential of tumor cells expressing aquaporin water channels. FASEB J 20: 1892-1894, 2006

19. Barabutis N and Schally AV: Knocking down gene expression for growth hormone-releasing hormone inhibits proliferation of human cancer cell lines. Br J Cancer 98: 1790-1796, 2008.

20. Jemal A, Siegel R, Xu J and Ward E: Cancer statistics, 2010. CA Cancer J Clin 60: 277-300, 2010.

21. Janssen-Heijnen ML and Coebergh JW: The changing epidemiology of lung cancer in Europe. Lung Cancer 41: 245-258, 2003.

22. Devesa SS, Bray F, Vizcaino AP and Parkin DM: International lung cancer trends by histologic type: male: female differences diminishing and adenocarcinoma rates rising. Int $\mathbf{J}$ Cancer 117: 294-299, 2005

23. Jiang Y: Aquaporin-1 activity of plasma membrane affects HT20 colon cancer cell migration. IUBMB Life 61: 1001-1009, 2009.

24. Otterbach F, Callies R, Adamzik M, Kimmig R, Siffert W, Schmid KW and Bankfalvi A: Aquaporin 1 (AQP1) expression is a novel characteristic feature of a particularly aggressive subgroup of basal-like breast carcinomas. Breast Cancer Res Treat 120: 67-76, 2010.

25. Pan H, Sun CC, Zhou CY and Huang HF: Expression of aquaporin-1 in normal, hyperplasic, and carcinomatous endometria. Int J Gynaecol Obstet 101: 239-244, 2008.

26. Hoque MO, Soria JC, Woo J, Lee T, Lee J, Jang SJ, Upadhyay S, Trink B, Monitto C, Desmaze C, et al: Aquaporin 1 is overexpressed in lung cancer and stimulates NIH-3T3 cell proliferation and anchorage-independent growth. Am J Pathol 168: 1345-1353, 2006.

27. Andersen P, Villingshøj M, Poulsen HS and Stockhausen MT: Improved response by co-targeting EGFR/EGFRvIII and Src family kinases in human cancer cells. Cancer Invest 27: 178-183, 2009.

28. Shankardas J, Patil RV and Vishwanatha JK: Effect of downregulation of aquaporins in human corneal endothelial and epithelial cell lines. Mol Vis 16: 1538-1548, 2010.

29. Yu JL, May L, Lhotak V, Shahrzad S, Shirasawa S, Weitz JI, Coomber BL, Mackman N and Rak JW: Oncogenic events regulate tissue factor expression in colorectal cancer cells: Implications for tumor progression and angiogenesis. Blood 105: 1734-1741, 2005

30. Amarzguioui M, Peng Q, Wiiger MT, Vasovic V, Babaie E, Holen T, Nesland JM and Prydz H: Ex vivo and in vivo delivery of anti-tissue factor short interfering RNA inhibits mouse pulmonary metastasis of B16 melanoma cells. Clin Cancer Res 12: 4055-4061, 2006.

31. Wang X, Wang M, Amarzguioui M, Liu F, Fodstad $\varnothing$ and Prydz H: Downregulation of tissue factor by RNA interference in human melanoma LOX-L cells reduces pulmonary metastasis in nude mice. Int J Cancer 112: 994-1002, 2004.

32. Steeg PS: Tumor metastasis: Mechanistic insights and clinical challenges. Nat Med 12: 895-904, 2006.

33. Chambers AF, Groom AC and MacDonald IC: Dissemination and growth of cancer cells in metastatic sites. Nat Rev Cancer 2: 563-572, 2002 .

34. Nguyen DX, Bos PD and Massagué J: Metastasis: From dissemination to organ-specific colonization. Nat Rev Cancer 9: 274-284, 2009. 
35. Yu C, Pan K, Xing D, Liang G, Tan W, Zhang L and Lin D Correlation between a single nucleotide polymorphism in the matrix metalloproteinase-2 promoter and risk of lung cancer. Cancer Res 62: 6430-6433, 2002.

36. Chetty C, Lakka SS, Bhoopathi P and Rao JS: MMP-2 alters VEGF expression via alphaVbeta3 integrin-mediated PI3K/AKT signaling in A549 lung cancer cells. Int J Cancer 127: 1081-1095, 2010.

37. Rojiani MV, Alidina J, Esposito N and Rojiani AM: Expression of MMP-2 correlates with increased angiogenesis in CNS metastasis of lung carcinoma. Int J Clin Exp Pathol 3: 775-781, 2010.

38. Xu J, Lamouille S and Derynck R: TGF-beta-induced epithelial to mesenchymal transition. Cell Res 19: 156-172, 2009.

39. Miyazono K: Transforming growth factor-beta signaling in epithelial-mesenchymal transition and progression of cancer. Proc Jpn Acad Ser B Phys Biol Sci 85: 314-323, 2009.
40. Massagué J: TGFbeta in cancer. Cell 134: 215-230, 2008.

41. ten Dijke P, Goumans MJ and Pardali E: Endoglin in angiogenesis and vascular diseases. Angiogenesis 11: 79-89, 2008.

42. Scagliotti GV, Selvaggi G, Novello S and Hirsch FR: The biology of epidermal growth factor receptor in lung cancer. Clin Cancer Res 10: 4227s-4232s, 2004.

43. Nicholson RI, Gee JM and Harper ME: EGFR and cancer prognosis. Eur J Cancer 37 (Suppl 4): S9-S15, 2001.

44. Mendelsohn J and Baselga J: The EGF receptor family as targets for cancer therapy. Oncogene 19: 6550-6565, 2000.

45. Zhang Z, Chen Z, Song Y, Zhang P, Hu J and Bai C: Expression of aquaporin 5 increases proliferation and metastasis potential of lung cancer. J Pathol 221: 210-220, 2010. 